\title{
Hospital pharmacy information centres and their role as suppliers of information
}

\author{
H. MCNulTY \\ B.Pharm., M.P.S., Ph.D. \\ Department of Drug Information, South Western Regional Health Authority
}

\begin{abstract}
Summary
The development of the drug information services within the hospital area is described from its origin in America. The services currently available in the NHS are outlined and a description of the type of enquiries handled is given.
\end{abstract}

'IN nearly all fields of science existing knowledge which could usefully be applied to daily life far exceeds that which is actually applied' (Herxheimer, 1976). This statement sums up the present situation in medicine and pharmacy and is particularly applicable to drug usage. The need for the improved use of drugs has been frequently expressed and this need is so great that it is likely that no single group will be able to satisfy the demands totally (Parish, 1976).

The aim of the meeting is to try to determine the extent to which the various possible contributors can help improve the application of knowledge. It is not possible to consider the information pharmacist in isolation because pharmacists have always been advisers on the safe and effective use of drugs, not only to doctors, but also to nurses, patients and other pharmacists. This role must therefore be discussed before that of the information pharmacist can become clear. The information needs of the prescribing physician is the subject under discussion and the author will restrict his comments to this particular user, although pharmacists also have a considerable contribution to make in improving the safe and effective use of drugs by nurses and patients.

\section{The Pharmacist}

The report of the working party on the Hospital Pharmaceutical Service (1970) noted that functions of the service included 'to assist in efficient prescribing by advising on the nature and properties of medicaments and upon the selection of the most suitable substances and the form in which they should be prescribed'.

The extension of ward pharmacy services has resulted in increasing numbers of enquiries of greater complexity from doctors and nurses to the pharmacist in many hospitals. Ward Pharmacy has also helped improve feedback of information from ward level.
Furthermore, pharmacists monitor prescriptions $\vec{\circ}$ for mistakes and interactions and advise the prescriber accordingly. As the number of drugs and $\vec{\omega}$ their relative toxicity increased, more information $\frac{\Omega}{8}$ was available and the need for an organized in-co formation service was recognized to support these 3 roles of the pharmacist. Facilities of the service were $\mathrm{c}$ naturally made available to doctors.

The advantages of a drug information service are $\omega$ many. It provides a readily accessible information $U$ store for all members of the health care team which

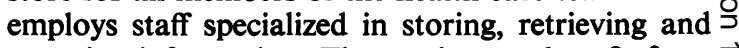
assessing information. The service can benefit from $\vec{C}$ co-ordination between centres to reduce duplication of effort and can justify the use of specialized equip- $\frac{\vec{D}}{\mathbb{D}}$ ment. Furthermore, the service offers personal cob 3 tact with local staff through the ward pharmacist or the general practice pharmacist, so that local de $\vec{\theta}$ mands can be met and specialist advisers at loca level can be consulted when clinical opinion $\bar{s}$. needed. Therefore, the development of an informa $\vec{a}-0$ tion network to feedback information and to communicate with other sources of information can become a reality.

\section{Development of the drug information service}

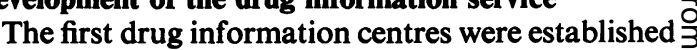
in America. The first centres in this country were $\supset$ established in 1970 in Leeds and London, and the Leeds centre became the first regional centre in the country in 1973. At the present time, there are 3 . special information posts at regional level in eleven regions in England, three in Scotland and one each?. in Northern Ireland and Wales. The structure within regions has developed according to local $₹$ demands, but most regions have a tiered network을 with centres at regional and/or area, district or local $>$ level, although these are staffed by pharmacists with을. dual responsibilities in many instances. Some regions $N$ have developed centres at area level, whereas others. ${ }^{\circ}$ favour a single regional centre.

The Regional Drug Information Pharmacists' Group was established in October 1975 to avoid duplication of effort between centres and to promoteo the development of a co-ordinated network. Coordination of effort has already taken place in the + ? fields of producing abstracts, adoption of specialized 
files such as drugs excreted in breast milk, and the group is developing a code of practice for application to all centres. However, at the present time, lack of resources are inhibiting development and it is therefore quite possible that many doctors may know little about the present service though in some parts of the country doctors in the community are now being contacted (McNulty, 1976).

The information problems facing the prescriber were outlined this morning and basically these may fall into several categories. Information is needed to allow the selection of appropriate therapy for the individual patient, who may be elderly or pregnant, or in renal failure. Having selected the appropriate therapy, if drugs are included, the correct dose must be given by the correct route. Side effects, interactions or toxic reactions may occur once the drug is in use and difficulties may arise when attempting to discontinue therapy. Other particular areas of uncertainty are in the determination of the place of new products and difficulties of maintaining awareness of new developments (Medico-Pharmaceutical Forum Report, 1975). Having defined a particular problem, considerable literature searching may be needed to obtain an answer. The feedback of information to the Committee on Safety of Medicines and the manufacturer about adverse effects of drugs is a further problem area (Report of the CSM, 1975).

\section{Services provided by drug information centres}

Many centres provide a current awareness service through the provision of a local bulletin on topics of general interest, frequently produced in consultation with local experts on such problems as drugs excreted in breast milk, interactions of drugs with oral contraceptives, new side effects and new drugs. Other centres supply abstracts of articles from journals on drug related topics to inform the user of potential hazards recently reported, new products which may be available, or the use of existing products for different preparations. Lectures are given on drug related topics for the same purpose.

The need for improved information on drugs at user level has lead to the development of ward notes schemes and the submission of information to drug and therapeutics committees. Some information pharmacists are also involved in research related to improving information services.

The passive role of answering more complex enquiries is an integral part of the information service. It has been said that pharmacists have no clinical training and this fact is largely accepted, though in some regions clinical pharmacy is developing, and many schools of pharmacy are making their courses more relevant to the practical situation.

Questions asked of the information service fall into two basic categories; those which require an opinion and those which need factual data. When clinical opinion as opposed to pharmaceutical opinion is needed, information pharmacists contact experts in the field, such as bacteriologists, pathologists or clinical pharmacologists, and when factual information requiring detailed searching is needed, the assistance of librarians and other information services is often sought. Perhaps the best method of illustrating the type of service offered is by the use of various examples. Elucidation of the true problem may need detailed questioning before an answer is attempted, so that the background of the question is understood and answers may require collation of information from various sources including books, journals and manufacturers. I will mention only the real problems here.

Most enquiries can be classified into a small number of headings, such as availability, clinical use, costs, dosage, administration, identification, interactions, incompatibilities, side effects, stability, or toxic reactions, and others.

Questions asked about availability include: 'Who supplies amiodarone required for a patient with Wolff-Parkinson-White syndrome?', or 'As vasopressin injection is not available what is available for a patient with bleeding oesophageal varices?' Questions relating to the clinical use of drugs include: 'Which antibiotic cream does not contain lanolin?', or 'Which drugs are available for treating night cramps?', or 'Which $\alpha$-adrenergic blocker is suitable for long-term treatment of phaeochromocytoma for a patient in whom an operation is contraindicated?'

Queries relating to the cost of therapy are selfexplanatory, but may relate to the cost of individual drugs or the cost of groups of drugs to the hospital or the National Health Service as a whole.

Dosage/administration queries may relate to 'The need for test doses before long-term iron injections', 'The intranasal administration of insulin', 'The crushing of methionine tablets for administration via a nasogastric tube', or 'The rate of administering amikacin injection intravenously'.

Identification questions include the identification of tablets or drug names. Questions of this type may be 'What is AH 5158?', or 'What type of drug is novodipam?'.

Enquiries relating to interactions of drugs with foods, or other drugs, are frequently asked, such as 'A patient taking a low dose oestrogen contraceptive had breakthrough bleeding when griseofulvin was commenced and ceased when a higher dose contraceptive was given. Is this a known interaction and what is the possible mechanism?' More comprehensive enquiries include: 'Which drugs interact with oral anticoagulants, and where possible give alternatives which do not interact'. 
Requests for information on incompatibility of drugs with intravenous fluids are also answered.

Questions relating to side effects of drugs are often of the nature where a patient has been on four or five drugs and has developed a side effect, such as agranulocytosis or acute renal failure, and the doctor requires to know which drug, if any, is responsible. Occasionally new side effects are suspected, such as bronchospasm with ibuprofen, and in these instances the manufacturers are usually contacted for up-todate information. If the side effect is noted, practitioners are encouraged to report their findings to the Committee on Safety of Medicines and the manufacturers.

Problems of stability of preparations and appropriate methods of storage are also answered.

Questions which relate to treatment of poisonings are usually referred to Poisons Information Centres, unless a specific hospital policy has been adopted, as may be the case with paracetamol overdosage.

Other problems which require answering cover a wide spectrum of categories including bioavailability, structural classification of drugs, legal problems, etc.

\section{Conclusion}

In conclusion, therefore, the need for a locally accessible central point for information on drugs and $\frac{3}{2}$ related problems is apparent. The service must be $\propto$ available locally, so that local contacts can be $\stackrel{\complement}{\leftarrow}$ developed and to allow cheap access via telephone $\overrightarrow{\vec{\rho}}$ for most enquirers. The information service offered by hospital pharmacists does not simply constitute an enquiry answering service, nor does it really act in a $\frac{\bar{c}}{\bar{c}}$ referral role, though such a role may also save the $\frac{\mathbb{D}}{\mathscr{D}}$ clinician time and effort.

It is interesting to note that in Australia steps are now being taken to develop similar services at State $\overrightarrow{0}$ level (Editorial, 1976).

\section{References}

Annual Report of the Committee on the Safety of Medicinese (1975) H.M. Stationery Office.

Editorial (1976) Where to turn? Australian Prescriber, 3, 45."

HerXHeImer, A. (1976) Educating doctors to use drugs well. $\omega$ British Journal of Clinical Pharmacology, 3, 111.

Report of the Working Party on the Continuing Education of $\mathrm{w}$ Doctors in Medicine and Therapeutics (1975) Medico-or Pharmaceutical Forum.

MCNulty, H. (1976) National network of centres needed.o Pharmaceutical Journal, 217, 454.

PARrish, P.A. (1976) Medicinal treatments: The future role $\overrightarrow{-}$ of the physician and pharmacist. Pharmaceutical Journal, $\mathbb{D}$ 217, 241.

Report of the Working Party of Hospital Pharmaceutical Services (1970) HM (70) 21 DHSS. 